\title{
Expression of vascular endothelial growth factor in uveal melanoma and its correlation with metastasis
}

\author{
Tom G Sheidow, Phil L Hooper, Cathy Crukley, Jean Young, J Godfrey Heathcote
}

\begin{abstract}
Aims-To evaluate the expression of vascular endothelial growth factor (VEGF) in uveal melanomas and correlate its presence with tumour characteristics and systemic metastasis.

Methods-47 cases of ciliochoroidal melanoma enucleated between 1983 and 1993 were retrieved from the pathology archives at the University of Western Ontario. Paraffin sections stained with haematoxylin and eosin, periodic acid Schiff, and periodic acid Schiff without haematoxylin after bleaching of melanin were examined. The expression of VEGF protein was examined by an immunoalkaline phosphatase method following antigen retrieval, using an antibody to VEGF and vector red as the chromogen. The intensity of VEGF immunoreactivity was graded on a scale of $0-7$ and correlated with tumour cell type, tumour size, presence or absence of necrosis, pigmentation, mitotic activity, microvascular density, and microvascular pattern.
\end{abstract}

Results-VEGF immunoreactivity was present in 44/47 tumours (94\%): the intensity was graded as very weak (1-2) in 29/47 $(62 \%)$ and as weak or greater in $15 / 47$ $(32 \%)$. VEGF was also found in the ciliary epithelium, smooth muscle of the ciliary body and iris, retinal ganglion cells, inner photoreceptor segments, and the retinal pigment epithelium. Follow up data were available in $43 / 47$ patients $(91.5 \%)$, with a median follow up time of 10 years. 16/43 (37\%) patients developed metastases. VEGF expression in melanoma was linked to the presence of tumour necrosis and the degree of pigmentation but no statistically significant relation with microvascular pattern, tumour size, or microvascular density was found. There was no statistically significant correlation between VEGF expression and metastasis.

Conclusions-Most ciliochoroidal melanomas express VEGF and expression is correlated with the presence of necrosis but not with the occurrence of systemic metastasis or tumour angiogenesis.

(Br F Ophthalmol 2000;84:750-756)

Tom Sheidow, Ivey Institute of Ophthalmology, 750 Commissioners Road E,

London, Ontario, N6A 4G5,

Canada

tgsheido@julian.uwo.ca

Accepted for publication 3 February 2000 has received significant attention in the recent literature as an angiogenic growth factor both in vivo and in vitro. ${ }^{1}$ It is a glycosylated, multifunctional cytokine, consisting of a family of dimeric polypeptides, which exerts its effects through specific receptors predominantly expressed on vascular endothelium. VEGF is not only involved in angiogenesis and endothelial cell growth but can also increase monocyte activation, chemotaxis, and vascular permeability. ${ }^{1}$

Many solid tumours have been found to express VEGF including adenocarcinoma of the gastrointestinal tract ${ }^{2}$ and breast, ${ }^{3}$ gliomas, ${ }^{4}$ and haemangioblastomas. ${ }^{5}$ Evidence for an important role for VEGF in tumour genesis was found by Kim et $a l^{6}$ who showed that the growth of experimental gliomas and rhabdomyosarcomas in vivo could be inhibited by neutralising antibody to VEGF.

The role of VEGF in melanoma growth and metastasis has been studied by immunohistochemical methods. Gitay-Goren et $a l^{7}$ looked at binding of VEGF to normal neonatal human melanocytes and four melanoma cell lines from both primary and metastatic melanomas cultured in vitro. These investigators found that human melanoma cells, but not normal melanocytes, express VEGF receptors, suggesting that the expression of VEGF receptors is one of the phenotypic changes occurring in melanocytes during malignant transformation. Salven et $a l^{8}$ found that $32 \%$ of primary cutaneous melanomas expressed cytoplasmic VEGF in $1-2 \%$ of the melanoma cells; VEGF expression was noted in $91 \%$ of melanoma metastases with an increased proportion of positive cells (0-60\%, mean $9 \%$ ).

The role of VEGF in tumour metastasis was explored $^{9}$ in a model in which melanoma cells were transfected with VEGF cDNA and then injected into immunodeficient mice. The expression of VEGF was associated with an increased tumour growth rate, increased tumour angiogenesis, decreased tumour necrosis, and an increase in experimental metastases. Cells expressing VEGF were found to form tumours with striking microvascular hyperpermeability and angiogenesis, leading to the suggestions that VEGF has an important role in facilitating tumour growth in vivo by stimulating angiogenesis. Potgens et $a l^{10}$ attempted to determine the expression of VEGF in cultured human melanoma cells and in a xenograft model. The degree of VEGF expression in vitro was increased in cultured cell lines with a known increased metastatic potential, but this trend was not observed in vivo. In another xenograft model, ${ }^{11}$ a correlation was found between VEGF mRNA and the degree of tumour vascularisation and the development of 
experimental metastasis. No correlation between vascular density and metastasis was found.

Immunohistochemical investigations into the expression of VEGF in ocular tissues have shown variable results. One study ${ }^{12}$ showed that VEGF is present at only very low levels in the retina and choroid of normal eyes. Kvanta et $a l^{13}$ showed that retinoblastomas contained high levels of both VEGF and VEGF mRNA, but were unable to demostrate either the protein or the mRNA in posterior uveal melanomas,. In contrast, Vinores et $a l^{14}$ found expression of VEGF within uveal melanomas, with $26 \%$ of tumours showing positivity. Expression of both VEGF mRNA and protein in ocular melanomas has been demonstrated by Stitt et al. ${ }^{15}$

The purpose of the current study was to examine further the expression of VEGF in uveal melanomas and to correlate its presence with tumour characteristics and systemic metastasis.

\section{Methods}

A total of 51 eyes enucleated at the University of Western Ontario between 1983 and 1993 for uveal melanoma were considered for this review. Forty seven pathological specimens could be retrieved from the pathology archives or had sufficient tumour left in the block for evaluation. No eyes assessed had received previous irradiation or thermotherapy. Routine sections were prepared and stained with haematoxylin and eosin, with and without bleaching, and periodic acid Schiff reagent without haematoxylin following bleaching.

ASSESSMENT OF TUMOUR CHARACTERISTICS

The pathology specimens were evaluated by two of the authors (JGH and TGS). Haematoxylin and eosin stained sections, with and without bleaching, were used to assess the degree of pigmentation (minimal, moderate, marked), cell type (epithelioid, spindle or mixed), and necrosis (absent or present). The tumour height and maximal basal diameter were measured from a glass slide using calipers with a Vernier scale. Mitoses were counted in $20 \mathrm{hpf}$ using a $\times 40$ objective with a field of $0.204 \mathrm{~mm}^{2}$. A bleached section of each tumour was stained with periodic acid Schiff (PAS) without haematoxylin for evaluation of the microvascular pattern; classification was based upon the patterns defined by Folberg et al. ${ }^{16}$ The tumours were categorised based on the absence or presence of vascular loops or networks.

Microvascular density was determined using an antibody to factor VIII with an immunoperoxidase technique according to the method of Foss et al. ${ }^{17}{ }^{18}$ Areas of high density (Fig 1(1)) were localised under $\times 100$ magnification and then counts were performed at a magnification of $\times 250$ power using a $0.175 \mathrm{~mm}$ square graticule. Three separate areas were counted for each tumour, with the average and maximum for each tumour recorded and converted into a count per $\mathrm{mm}^{2}$.
IMMUNOHISTOCHEMICAL STAINING FOR VEGF Slides of each specimen were deparaffinised and hydrated through graded alcohols. For antigen retrieval, sections were placed in Coplin jars containing $10 \mathrm{mM}$ citrate buffer, $\mathrm{pH}$ 6.0, and heated in a microwave on high for 3.5 minutes, allowing the buffer to boil, followed by 10 minutes at medium low. After cooling, the slides were washed briefly in water and incubated in prewarmed $0.015 \%$ trypsin at $37^{\circ} \mathrm{C}$ for 45 minutes. Carazzi's haematoxylin was applied as counterstain and the slides incubated with normal goat serum at room temperature for 30 minutes. Slides were incubated overnight at $4^{\circ} \mathrm{C}$ with VEGF A-20 antibody (Rabbit IgG, Santa Cruz Biotechnology, Inc, Santa Cruz, CA, USA), at a dilution of $1: 2000$. After washing with phosphate buffered saline (PBS), $\mathrm{pH} 7.6$, slides were further incubated with biotinylated secondary antibody (goat anti-rabbit IgG, Vector Burlingame, CA, USA) for 45 minutes, washed as before with PBS, and treated for 40 minutes with dilute Vectastain ABC-AP reagent, prepared according to the manufacturer's instructions. After a final wash the slides were incubated with freshly prepared alkaline phosphatase substrate solution (vector red), containing one drop of $125 \mathrm{mM}$ levamisole to inhibit endogenous alkaline phosphatase, for 20 minutes before washing with water, dehydrating quickly through graded alcohols, and clearing with xylene. As a negative control, a slide of each tumour was incubated with normal rabbit serum (Rabbit Immunoglobulin Fraction, Dako, Dimension Laboratories, Mississauga, Ontario, Canada) instead of the anti-VEGF antibody. There was no evidence of imunoreactivity in the control slides.

The selection of the appropriate dilution of antibody was based upon evaluation of human kidney specimens (Fig 1(2)) and eyes exposed to various dilutions. Higher concentrations were found to stain heavily several cell types in renal tissue while a 1:2000 dilution appeared to react only in the proximal convoluted tubules (weak) and in the collecting ducts (strong). Specificity of the antibody was confirmed by pre-absorption with blocking peptide (VEGF peptide, SC152P, Santa Cruz Biotechnology Inc) according to the manufacturer's instructions. The use of the blocking peptide eliminated the immunohistochemical demonstration of VEGF in the renal tubules, retinal cells, non-pigmented ciliary epithelium, ciliary muscle, and melanoma cells.

ASSESSMENT OF VEGF IMMUNOHISTOCHEMICAL STAINING

VEGF immunoreactivity was graded independently by two observers (JGH, TGS) from 0 to 7 as outlined by Lutty et $a l^{12}$ (Table 1). Disagreement between observers was resolved by joint examination of the slides in question and establishment of a mutually agreeable grade. Uniform staining was defined in our study as the presence of reaction product in virtually all areas of the tumour, except for areas of necrosis, when the slide was examined at low power. The presence of reaction product within the 

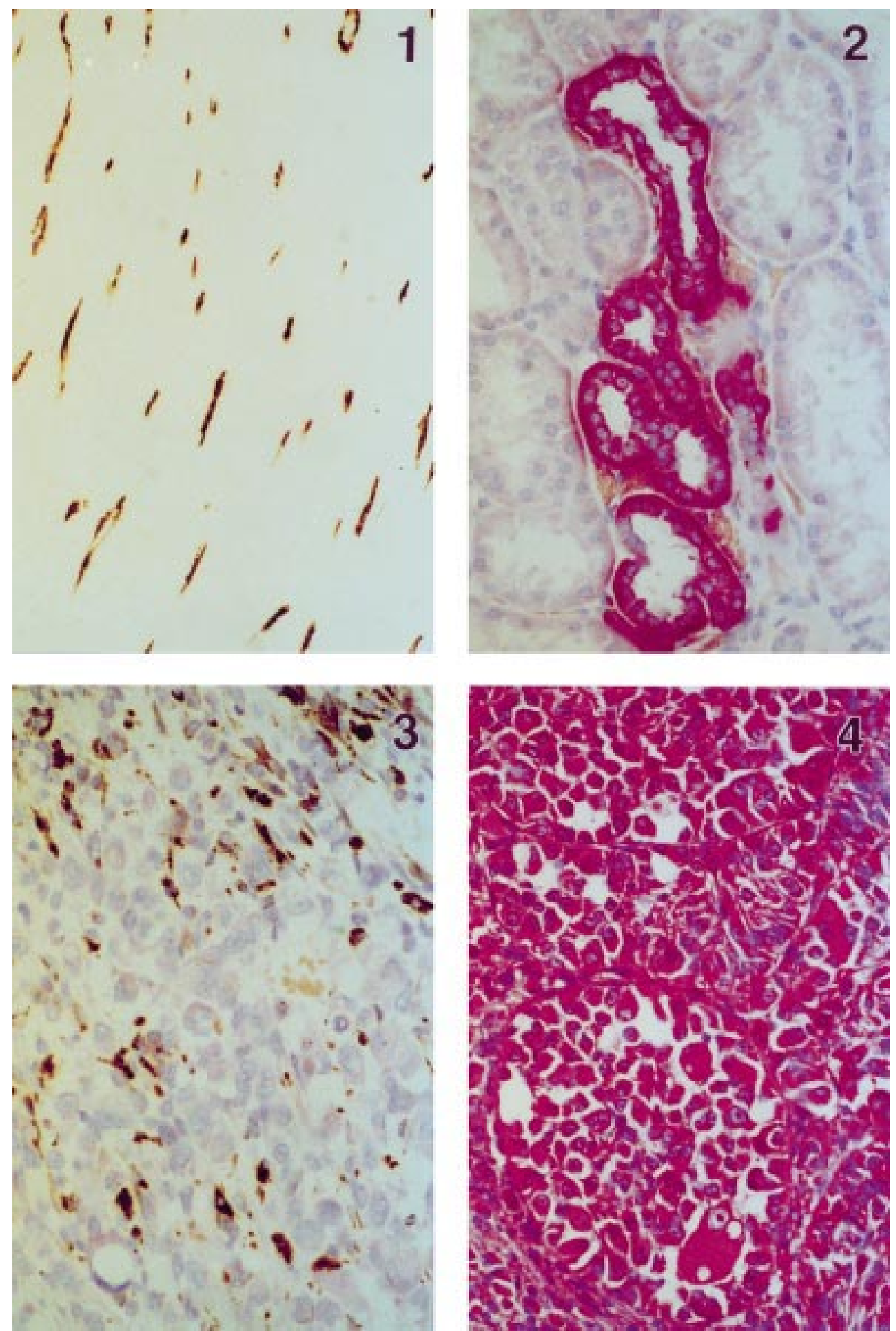

Figure 1 (1) Immunoperoxidase demonstration of factor VIII expression in a choroidal melanoma with high microvascular density $(\times 250)$. (2) Immunoalkaline phosphatase demonstration of VEGF expression in the collecting ducts (moderate) and convoluted tubules (weak) of human kidney, using vector red as chromogen and anti-VEGF antibody at a dilution of 1:2000 ( $\times 400)$. (3) VEGF expression (grade 2, very weak and uniform) in epithelioid cells of a choroidal melanoma (×400). (4) VEGF expression (grade 6, moderate and uniform) in epithelioid cells of a choroidal melanoma.

The outline of a microvascular network can be seen $(\times 400)$.

non-pigmented ciliary epithelium, retinal ganglion cells, and ciliary/iris musculature acted as an internal, positive control for each specimen.

Data analysis was performed on the sample data utilising logistic regression for the determination of tumour characteristic correlations with metastases. Odds ratios and $\mathrm{p}$ values were calculated for each characteristic utilising a $\chi^{2}$ model with 1 degree of freedom. To assess the ability of VEGF to predict other tumour characteristics and the development of metastases, logistic regression and polychotomous regression analyses were applied as appropriate, based on the outcome variable. 
Table 1 Grading scale for the expression of VEGF in uveal melanomas

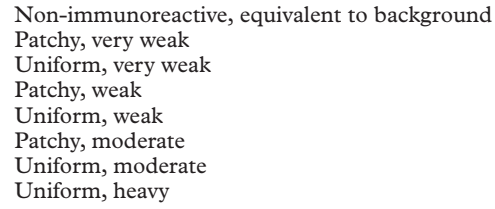

\section{Results}

Of 51 eyes containing uveal melanomas enucleated between 1983 and 1993, archival pathology specimens were available for 47 $(88 \%)$. Four specimens could not be located or had insufficient tumour remaining for evaluation. The mean patient age at the time of enucleation was 62.1 years; $25(53.2 \%)$ of the patients were male and $22(46.8 \%)$ female. All patients underwent rigorous medical evaluation to exclude systemic metastases before enucleation. The mean follow up time was 9.9 years (range 5 to 15 years). Follow up information was obtained for 43/47 (91.5\%) patients and within this group 16/43 (37.2\%) were diagnosed with systemic metastases. Further evaluation revealed that of the 22 patients with less than 10 years of follow up, six
$(27.3 \%)$ patients had developed metastases, while of the 21 patients with greater than 10 years of follow up, $10(47.6 \%)$ had developed metastases.

Tumour and patient characteristics are presented in Table 2 . The melanomas evaluated included $18(38.3 \%)$ spindle cell, 24 $(51.1 \%)$ mixed cell, and five $(10.6 \%)$ epithelioid cell tumours. Necrosis was found to be present in 20/47 (42.6\%). Pigmentation was graded as minimal in $21 / 47(44.7 \%)$, moderate in $15 / 47(31.9 \%)$, and marked in $11 / 47$ $(23.4 \%)$. The number of mitotic figures per 20 hpf was greater than 10 in $13 / 47(27.6 \%)$. Tumour size was graded as small (less than 10 $\mathrm{mm}$ in maximum dimension) in 21/47 $(44.7 \%)$, medium ( $>10 \mathrm{~mm}$ and $<15 \mathrm{~mm}$ ) in $20 / 47(42.6 \%) \mathrm{m}$ and large (15 $\mathrm{mm}$ or greater) in $6 / 47(12.8 \%)$. Microvascular loops and/or networks were seen in $18 / 46(39.1 \%)$ of the tumours with the remaining $28 / 46(59.6 \%)$ tumours displaying one or more of the other patterns described by Folberg et al. ${ }^{16}$

VEGF scores were obtained for all 47 melanomas and a positive reaction was present in $44 / 47(93.6 \%)$. The VEGF score was very weak ( 1 or 2 ) in $29 / 47(61.7 \%$ ), as depicted in Figure 1(3), and weak or moderate (3 or

Table 2 Tumour and patient characteristics

\begin{tabular}{|c|c|c|c|c|c|c|c|c|c|c|c|}
\hline \multirow[b]{2}{*}{ Year } & \multirow[b]{2}{*}{ Age } & \multirow[b]{2}{*}{$\operatorname{Sex}$} & \multirow[b]{2}{*}{ Cell type } & \multirow[b]{2}{*}{ Pigmentation } & \multirow[b]{2}{*}{ Necrosis } & \multirow[b]{2}{*}{ Mitoses * } & \multirow[b]{2}{*}{ Size } & \multicolumn{2}{|c|}{ Microvascular } & \multirow[b]{2}{*}{$V E G F$} & \multirow[b]{2}{*}{ Metastases } \\
\hline & & & & & & & & Pattern & Densityt & & \\
\hline 1987 & 41 & $\mathrm{~F}$ & Spindle & Minimal & Absent & 6 & 6.1 & Other & 98 & 2 & No \\
\hline 1990 & 53 & $\mathrm{~F}$ & Mixed & Minimal & Present & 6 & 13.6 & Other & 294 & 4 & Yes \\
\hline 1990 & 64 & M & Spindle & Marked & Present & 1 & 4.2 & Other & 65 & 2 & No \\
\hline 1991 & 72 & M & Spindle & Minimal & Present & 2 & 12.7 & Other & 33 & 4 & No \\
\hline 1983 & 65 & $\mathrm{~F}$ & Mixed & Marked & Absent & 0 & 8.1 & Other & 163 & 1 & No \\
\hline 1985 & 84 & $\mathrm{~F}$ & Spindle & Marked & Absent & 1 & 10.4 & Other & 65 & 1 & No \\
\hline 1989 & 78 & $\mathrm{M}$ & Mixed & Moderate & Present & 13 & 12.4 & Network & 392 & 4 & Lost \\
\hline 1986 & 74 & M & Epithelioid & Marked & Absent & 10 & 15.3 & Network & 490 & 1 & Yes \\
\hline 1987 & 84 & $\mathrm{M}$ & Mixed & Moderate & Absent & 11 & 11.6 & Network & 196 & 1 & Lost \\
\hline 1988 & 41 & $\mathrm{~F}$ & Mixed & Minimal & Absent & 12 & 8.0 & Network & 196 & 2 & Yes \\
\hline 1986 & 29 & M & Mixed & Marked & Absent & 10 & 12.6 & Other & 261 & 1 & Yes \\
\hline 1992 & 88 & $\mathrm{~F}$ & Mixed & Minimal & Present & 10 & 9.5 & Loop & 98 & 1 & No \\
\hline 1987 & 71 & $\mathrm{~F}$ & Mixed & Moderate & Present & 7 & 10.2 & Network & 163 & 1 & Lost \\
\hline 1987 & 67 & $\mathrm{M}$ & Mixed & Moderate & Absent & 2 & 14.6 & Network & 131 & 0 & Yes \\
\hline 1987 & 75 & M & Mixed & Minimal & Absent & 5 & 7.2 & Other & 327 & 2 & No \\
\hline 1991 & 42 & M & Spindle & Minimal & Absent & 2 & 10.0 & Other & 33 & 2 & No \\
\hline 1987 & 67 & M & Mixed & Marked & Absent & 3 & 7.0 & Other & 294 & 0 & No \\
\hline 1986 & 71 & M & Mixed & Moderate & Present & 16 & 11.9 & Network & 359 & 2 & Yes \\
\hline 1990 & 39 & M & Spindle & Minimal & Absent & 2 & 13.3 & Other & 33 & 3 & No \\
\hline 1989 & 56 & $\mathrm{~F}$ & Epithelioid & Minimal & Present & 1 & 15.5 & Loop & 131 & 6 & Yes \\
\hline 1989 & 78 & $\mathrm{~F}$ & Spindle & Minimal & Present & 8 & 8.7 & Other & 65 & 2 & No \\
\hline 1986 & 50 & $\mathrm{~F}$ & Epithelioid & Marked & Absent & 10 & 10.0 & Other & 131 & 2 & Yes \\
\hline 1986 & 72 & M & Spindle & Minimal & Absent & 0 & 7.7 & Other & 65 & 1 & No \\
\hline 1992 & 35 & $\mathrm{M}$ & Mixed & Marked & Absent & 2 & 6.6 & Loop & 261 & 6 & No \\
\hline 1983 & 58 & M & Mixed & Minimal & Absent & 8 & 9.8 & Other & 261 & 2 & No \\
\hline 1986 & 75 & M & Spindle & Moderate & Present & 3 & 14.2 & Other & 33 & 1 & No \\
\hline 1985 & 50 & $\mathrm{~F}$ & Spindle & Moderate & Absent & 0 & 9.3 & Other & 65 & 1 & No \\
\hline 1992 & 77 & $\mathrm{~F}$ & Spindle & Minimal & Absent & 1 & 9.6 & Other & 229 & 3 & No \\
\hline 1987 & 19 & $\mathrm{M}$ & Epithelioid & Minimal & Present & 7 & 8.4 & Network & 98 & 6 & Yes \\
\hline 1990 & 66 & $\mathrm{~F}$ & Mixed & Minimal & Absent & 7 & 4.9 & Other & 65 & 2 & No \\
\hline 1991 & 61 & M & Spindle & Minimal & Present & 34 & 12.4 & Other & 131 & 4 & No \\
\hline 1990 & 64 & M & Spindle & Moderate & Present & 16 & 14.8 & Other & 65 & 4 & No \\
\hline 1990 & 23 & $\mathrm{~F}$ & Spindle & Minimal & Absent & 5 & 4.4 & Other & 98 & 2 & No \\
\hline 1987 & 44 & M & Epithelioid & Minimal & Present & 24 & 17.5 & Loop & 914 & 4 & Yes \\
\hline 1989 & 44 & M & Mixed & Minimal & Absent & 4 & 7.9 & Other & 98 & 2 & No \\
\hline 1992 & 65 & M & Mixed & Moderate & Absent & 4 & 12.9 & Loop & 131 & 3 & Yes \\
\hline 1990 & 71 & $\mathrm{~F}$ & Mixed & Minimal & Absent & 5 & 9.3 & Other & 196 & 2 & Yes \\
\hline 1989 & 75 & M & Mixed & Moderate & Absent & 24 & 13.9 & Loop & 98 & 1 & Yes \\
\hline 1987 & 78 & $\mathrm{~F}$ & Spindle & Moderate & Present & 6 & 10.0 & Loop & 163 & 2 & Yes \\
\hline 1985 & 34 & $\mathrm{~F}$ & Spindle & Moderate & Present & 2 & 12.4 & Other & 98 & 1 & Yes \\
\hline 1984 & 75 & $\mathrm{~F}$ & Mixed & Marked & Present & 18 & 15.0 & Network & 327 & 1 & No \\
\hline 1987 & 67 & M & Mixed & Marked & Absent & 0 & 4.7 & Other & 131 & 2 & No \\
\hline 1987 & 50 & $\mathrm{~F}$ & Spindle & Moderate & Present & 5 & 7.4 & Network & 163 & 3 & Lost \\
\hline 1992 & 88 & $\mathrm{~F}$ & Mixed & Marked & Absent & 2 & 18.0 & & 65 & 4 & No \\
\hline 1990 & 80 & $\mathrm{~F}$ & Mixed & Minimal & Present & 9 & 15.2 & Network & 327 & 4 & No \\
\hline 1992 & 27 & $\mathrm{~F}$ & Moderate & Moderate & Present & 4 & 5.0 & Other & 65 & 1 & No \\
\hline 1987 & 69 & M & Spindle & Moderate & Absent & 3 & 14.8 & Other & 33 & 0 & Yes \\
\hline
\end{tabular}


Table 3 Relation of tumour characteristics to metastases and $V E G F$

\begin{tabular}{|c|c|c|}
\hline & Metastasis & Mean VEGF \\
\hline \multicolumn{3}{|l|}{ Cell type ${ }^{\star}$} \\
\hline Spindle & $3 / 17(17.6 \%)$ & 2.11 \\
\hline Mixed & $8 / 21(38.1 \%)$ & 2.04 \\
\hline Epithelioid & $5 / 5(100 \%)$ & 3.8 \\
\hline \multicolumn{3}{|l|}{ Size $^{\star}$} \\
\hline Small & $3 / 20(15 \%)$ & 2 \\
\hline Medium & $10 / 17(58.8)$ & 2.16 \\
\hline Large & $3 / 6(50 \%)$ & 3.33 \\
\hline \multicolumn{3}{|l|}{ Mitoses* } \\
\hline $0-9$ per $20 \mathrm{hpf}$ & $9 / 32(28.2 \%)$ & 2.29 \\
\hline$>10$ per $20 \mathrm{hpf}$ & $7 / 11(63.6 \%)$ & 2.15 \\
\hline \multicolumn{3}{|l|}{ Necrosist } \\
\hline Absent & $9 / 26(34.6 \%)$ & 1.81 \\
\hline Present & $7 / 17(41.2)$ & 2.85 \\
\hline \multicolumn{3}{|l|}{ Pigmentation $\dagger$} \\
\hline Minimal & $6 / 21(28.6 \%)$ & 2.86 \\
\hline Moderate & $7 / 11(63.4 \%)$ & 1.67 \\
\hline Marked & $3 / 11(27.3 \%)$ & 1.91 \\
\hline \multicolumn{3}{|c|}{ Microvascular pattern ${ }^{\star}$} \\
\hline Other & $6 / 28(21.4 \%)$ & 1.93 \\
\hline Loop/networks & $10 / 14(71.4 \%)$ & 2.78 \\
\hline \multicolumn{3}{|c|}{ Microvascular density } \\
\hline$<75 / \mathrm{mm}^{2}$ & $1 / 14(7.1 \%)$ & 2 \\
\hline $75-150 / \mathrm{mm}^{2}$ & $7 / 13(53.8 \%)$ & 2.46 \\
\hline $150-225 / \mathrm{mm}^{2}$ & $3 / 4(75.0 \%)$ & 1.71 \\
\hline$>225 / \mathrm{mm}^{2}$ & $5 / 12(41.7 \%)$ & 4.85 \\
\hline
\end{tabular}

${ }^{\star}$ Correlation with metastasis.

+Correlation with VEGF.

greater) in $15 / 47(31.9 \%)$, as seen in Figure $1(4)$. The mean VEGF score for all melanomas combined was 2.25 .

The results in Table 3 present the VEGF scores and the occurrence of metastases for each of the tumour characteristics. Tumour cell type, mitotic count, tumour size, and microvascular pattern were all correlated with metastasis at a statistically significant level. A trend towards a positive correlation with VEGF score was seen with the presence of necrosis, microvascular pattern, and tumour size; however, only the presence of necrosis and the degree of pigmentation achieved statistical significance. Within the group that had developed metastases, the mean VEGF score was 2.31 while in the group that had not developed metastases the mean VEGF score 2.2. This difference was not statistically significant.

Within our specimens, VEGF immunoreactivity was present consistently in many normal ocular tissues including the ciliary epithelium, which was used as a positive internal control for the study, RPE, ganglion cells, photorecep-

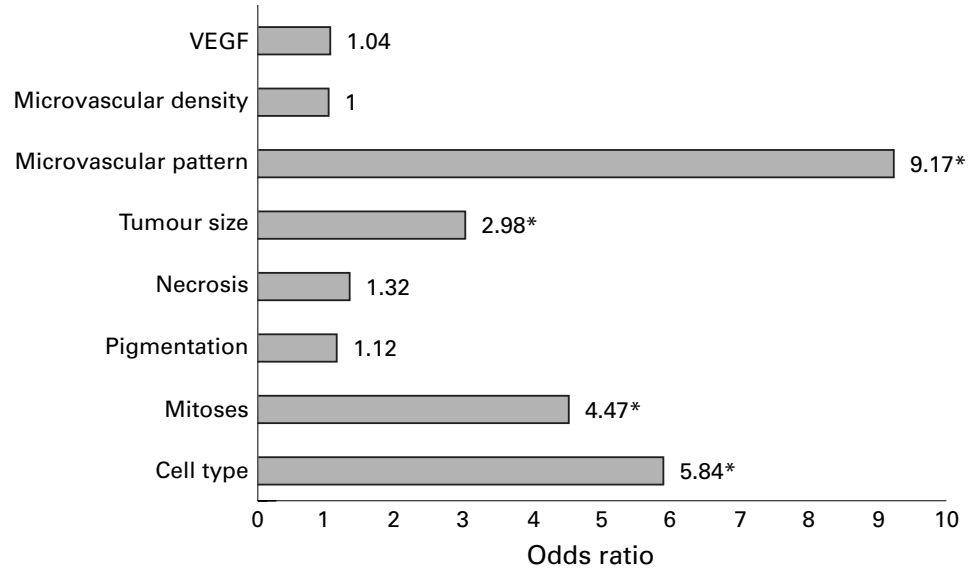

Figure 2 Odds ratios for the development of metastases. Logistic regression analysis for each tumour characteristic and its relation to the risk of development of metastases. An asterisk indicates a statistically significant result. tor inner segments, and the musculature of the ciliary body and iris.

The results of the statistical analyses depicting the odds ratios for the development of metastases for each tumour characteristic are shown in Figure 2. Tumour size, cell type, number of mitotic figures and microvascular pattern were found to have statistically significant odds ratios.

\section{Discussion}

VEGF expression has been well documented in several solid tumours ${ }^{2-5}$ and in non-ocular melanoma cells. ${ }^{78}$ Attempts to demonstrate VEGF in ocular melanomas by immunohistochemistry have yielded inconsistent results. ${ }^{131419}$ Kvanta et $a l^{13}$ looked at VEGF protein expression by immunohistochemical methods and mRNA levels by in situ hybridisation in eyes with retinoblastoma and posterior uveal melanoma. In retinoblastomas, 9/10 showed high levels of VEGF mRNA and 8/10 showed strong immunoreactivity; none of the melanomas showed high levels of either mRNA or protein. This difference was attributed to the high vascularity and rapid growth rate of retinoblastoma compared with uveal tumours. Similarly, a study of VEGF in central retinal vein occlusion (CRVO), ${ }^{19}$ included five eyes with melanomas in the control group and found no VEGF immunoreactivity.

Vinores et $a l^{14}$ examined 39 eyes with melanoma for VEGF immunoreactivity and demonstrated VEGF in the retina in $46 \%$ of the eyes. The immunoreactivity was localised to the cells within the vessel walls of the inner retina, ganglion cells, photoreceptors, RPE, ciliary body, and iris. VEGF positivity was not correlated with specific pathological features of the specimen, including haemorrhage, exudative retinal detachment, tumour necrosis, size, invasiveness, or lymphocytic infiltration. Immunolocalisation of the staining within the tumour was inconsistent, with $26 \%$ of tumours showing a positive reaction within the centre of the tumour and $51 \%$ at the periphery.

More recent work by Stitt et $a l^{15}$ has demonstrated VEGF mRNA and protein in both ocular melanomas and retinoblastomas. In this study, VEGF expression was concentrated around blood vessels within the retinoblastomas but was more diffuse within the melanomas, a feature they attributed to the greater perfusion of the choroidal vasculature.

In this study we have attempted to address the controversy regarding the presence or absence of VEGF in uveal melanomas and to correlate this with known prognostic indicators as well as clinical follow up data. Our study has shown the presence of VEGF protein in the majority of melanomas. Only 3/47 (6.4\%) showed no VEGF protein while weak or moderate levels ( 3 or greater) were present in 15/47 $(31.9 \%)$. Figure $1(3)$ represents a VEGF grade 2 (very weak, uniform) and clearly depicts the presence of cytoplasmic reaction product. In grading the strength of the reaction product the periphery of the tumour was ignored since immunohistochemical reactions tend to be more intense at the edge of a tissue sample. 
This technical artefact may explain the increased VEGF positivity previously noted in the periphery of the tumours. ${ }^{14}$ The majority of specimens in our study (26/43-60\%) showed VEGF positivity uniformly throughout the tumour rather than localised to specific areas, in agreement with the results of Stitt et al. ${ }^{15}$ Although the melanomas included in this study represent archival pathology specimens, similar investigations into the expression of VEGF protein in ocular melanomas (I Cree, personal communication) have not shown a correlation between VEGF and the age of the specimen.

Despite VEGF protein levels being statistically correlated with the presence of necrosis there was no recognisable increase in VEGF expression in areas adjacent to necrosis. Necrosis within the tumours indicates some degree of tumour ischaemia and results in hypoxia induced expression of VEGF, a well documented characteristic of VEGF. This correlation between VEGF and necrosis is consistent with previous studies ${ }^{9}$ showing that the increased expression of VEGF in melanoma cells was associated with less tumour necrosis.

Recent work ${ }^{12}$ has suggested that the normal human retina does not express VEGF but VEGF is present in the diabetic retina, possibly as a result of hypoxia which is known to increase expression of the growth factor. In that study, five normal necropsy eyes were evaluated and VEGF immunoreactivity was graded as very weak and patchy (grade 1) or absent in four of five specimens. Vinores et $a l^{14}$ localised VEGF in all the layers of the retina as well as the ciliary body, iris, and retinal pigment epithelium in the cases of uveal melanoma but found that only one of 14 normal necropsy eyes had weak VEGF positivity in the retina and three of 14 demonstrated weak VEGF staining in the ciliary body. Other studies have shown VEGF mRNA and receptors present in all vascularised ocular tissues ${ }^{20}$ and that VEGF is expressed in all three nuclear layers of the retina in patients with CRVO. ${ }^{19}$ Stitt et $a l^{15}$ found an upregulation of VEGF mRNA and peptide expression in the retina but the levels of mRNA were high compared with the peptide, raising the possibility of some form of post-transcriptional regulation of VEGF expression.

In our study, examination of eyes containing melanomas revealed moderate to strong expression of VEGF protein in retinal ganglion cells and photoreceptor elements. Only occasional perikarya in the inner and outer nuclear layers demonstrated reaction product. The reaction was not restricted to the tissue adjacent to the tumour but appeared to be widespread within the retina in most specimens. In addition, immunoreactivity was seen in all cases in the non-pigmented ciliary epithelium and the smooth muscle of the ciliary body and iris.

The immunohistochemical demonstration of VEGF in our study used methods that are generally similar to those employed by Stitt et $a l^{15}$ but differ from the other studies ${ }^{13}{ }^{14}$ in sev- eral ways. Firstly, the antibody dilution was significantly greater (1:2000 for our study compared with 1:25, Kvanta et $a l^{13}$ and 1:20, Vinores et $\left.a l^{14}\right)$. The likelihood of spurious immunoreactivity at this dilution is considerably lower. Secondly, the sensitivity of our technique was enhanced by antigen retrieval (microwave treatment and trypsin digestion) and the use of an immunoalkaline phosphatase method with a red chromogen. The blocking of the immunohistochemical reaction by preincubation of the antibody with VEGF peptide confirmed the specificity of the immunostain.

Our study has attempted to answer the question of whether VEGF expression correlates with tumour metastasis. Previous studies ${ }^{10}$ have found the level of VEGF expression correlated with metastatic potential in melanoma cell lines in vitro but not in vivo. Melanoma VEGF expression and mRNA expression have been associated with increased tumour growth, increased tumour vascular density, and decreased necrosis as well as an increase in experimental metastases. ${ }^{9} 11$

In our study we were unable to establish a correlation between VEGF immunoreactivity and either microvascular pattern or microvascular density, both indicators of angiogenesis, nor could VEGF be correlated with the occurrence of metastasis in individual cases of uveal melanoma. Examination of the relation of VEGF expression and many factors known to be prognostic for metastasis revealed a relation only with the presence of tumour necrosis and the degree of pigmentation. Of interest, microvascular density was not found to be predictive of metastases in our study in contrast with previous work by Foss et $a l^{17}$ and Makitie et al. ${ }^{21}$

Our specimens appear to be representative of the general melanoma population with regard to the distribution of cell types, although very few large tumours were included in the study. The number of patients developing metastases is slightly lower than in some previous reports and may be related to the tumour size or to the shorter duration of follow up. In the group of patients with follow up available for more than 10 years, $11 / 22(50 \%)$ developed metastasis.

In conclusion, uveal melanomas have been shown to express VEGF protein although this expression could not be correlated with metastatic potential. VEGF was associated with the development of necrosis within tumours but did not correlate with markers of angiogenesis.

Special thanks to Jackie Williams (Department of Ophthalmology) and Larry Stitt (Biostatistical Support Unit, Department
of Epidemiology and Biostatistics, University of Western Ontario) for their assistance with the statistical analyses in this study.

1 Schlingemann RO, van Hinsbergh VWM. Role of vascular permeability factor/vascular endothelial growth factor in eye disease. Br $\mathcal{F}$ Ophthalmol 1997;81:501-12.

2 Brown LF, Berse B, Jackman RW, et al. Expression of vascular permeability factor (vascular endothelial growth factor) lar permeability factor (vascular endothelial growth factor)
and its receptors in adenocarcinomas of the gastrointestinal

3 Brown LF, Berse B, Jackman RW, et al. Expression of vascular permeability factor (vascular endothelial growth factor) and its receptors in breast cancer. Hum Pathol 1995;26: $86-91$. 
4 Samoto K, Ikezaki K, Ono M, et al. Expression of vascular endothelial growth factor and its possible relation with neovascularization in human brain tumors. Cancer Res 1995;55:1189-93.

5 Wizigmann-Voos S, Breier G, Risau W, et al. Up-regulation of vascular endothelial growth factor and its receptors in von Hippel-Lindau disease-associated and sporadic hemangioblastomas. Cancer Res 1995;55:1358-64.

$6 \mathrm{Kim} \mathrm{KJ}, \mathrm{Li} \mathrm{B}$, Winer J, et al. Inhibition of vascular endothelial growth factor-induced angiogenesis suppresses tumour growth in vivo. Nature 1993;362:841-4.

7 Gitay-Goren H, Halaban R, Neufeld G. Human melanoma cells but not normal melanocytes express vascular endothelial growth factor receptors. Biochem Biophys Res Commun 1993;190:702-8.

8 Salven P, Heikkila P, Joensuu H. Enhanced expression of vascular endothelial growth factor in metastatic melanoma. Br f Cancer 1997;76:930-4

9 Claffey KP, Brown LF, del AL, et al. Expression of vascular permeability factor/vascular endothelial growth factor by permeability factor/vascular endothelial growth factor by melanoma cells increases tumor growth, angiogenesis,

10 Potgens AJ, Lubsen NH, van AM, et al. Vascular permeability factor expression influences tumor angiogenesis in human melanoma lines xenografted to nude mice. $A m \mathcal{F}$ Pathol 1995;146:197-209.

11 Westphal JR, van't Hullenaar RG, van der Laak JA, et al. Vascular density in melanoma xenografts correlates with vascular permeability factor expression but not with metastatic potential. Br f Cancer 1997;76:561-70.

12 Lutty GA, McLeod DS, Merges C, et al. Localization of vascular endothelial growth factor in human retina and choroid. Arch Ophthalmol 1996;114:971-7.
13 Kvanta A, Steen B, Seregard S. Expression of vascular endothelial growth factor (VEGF) in retinoblastoma but not in posterior uveal melanoma. Exp Eye Res 1996;63: 511-18.

14 Vinores SA, Kuchle M, Mahlow J, et al. Blood-ocular barrier breakdown in eyes with ocular melanoma. A potential role for vascular endothelial growth factor/vascular permeability factor. Am F Pathol 1995;147:1289-97.

15 Stitt AW, Simpson DA, Boocock C, et al. Expression of vascular endothelial growth factor (VEGF) and its receptors is regulated in eyes with intra-ocular tumours. F Pathol 1998; 186:306-12.

16 Folberg R, Rummelt V, Parys-Van GR, et al. The prognostic value of tumor blood vessel morphology in primary uveal melanoma. Ophthalmology 1993;100:1389-98.

17 Foss AJ, Alexander RA, Jefferies LW, et al. Microvessel count predicts survival in uveal melanoma. Cancer Res 1996;56:2900-3.

18 Foss AJ, Alexander RA, Jefferies LW, et al. Immunohistochemical techniques:the effect of melanin bleaching [see comments]. Br f Biomed Sci 1995;52:22-5.

19 Pe'er J, Folberg R, Itin A, et al. Vascular endothelial growth factor upregulation in human central retinal vein occlusion. Ophthalmology 1998;105:412-16.

20 Kim I, Ryan AM, Rohan R, et al. Constitutive expression of VEGF, VEGFR-1, and VEGFR-2 in normal eyes. Invest Ophthalmol Vis Sci 1999;40:2115-21.

21 Makitie T, Summanen P, Tarkkanen A, et al. Microvascular density in predicting survival of patients with choroidal and ciliary body melanoma. Invest Ophthalmol Vis Sci 1999;40: 2471-80. 